\title{
Variance of Lipschitz functions and an isoperimetric problem for a class of product measures
}

\author{
SERGEI G. BOBKOV ${ }^{1}$ and CHRISTIAN HOUDRÉ ${ }^{2 *}$ \\ ${ }^{1}$ Department of Mathematics, Syktyvkar University, 167001 Syktyvkar, Russia \\ ${ }^{2}$ Center for Applied Probability, School of Mathematics, Georgia Institute of Technology, Atlanta, GA \\ 30322, USA
}

The maximal variance of Lipschitz functions (with respect to the $\ell^{1}$-distance) of independent random vectors is found. This is then used to solve the isoperimetric problem, uniformly in the class of product probability measures with given variance.

Keywords: isoperimetry; Lipschitz function; variance inequality

\section{Statements}

Let $\xi=\left(\xi_{1}, \ldots, \xi_{n}\right)$ be a vector of independent random variables with finite variance $\sigma_{i}^{2}=\operatorname{var} \xi_{i}, 1 \leq i \leq n$. Denote by $\mathscr{F}_{1}$ the class of all functions on $\mathbb{R}^{n}$ which are Lipschitz with respect to the $\ell^{1}$-distance

$$
d_{1}(x, y)=\|x-y\|_{1}=\sum_{k=1}^{n}\left|x_{k}-y_{k}\right|, \quad x, y \in \operatorname{R}^{n} .
$$

By definition, $f \in \mathscr{F}_{1}$, if for all $x, y \in \mathbb{R}^{n},|f(x)-f(y)| \leq d_{1}(x, y)$. Let $S_{n}=\xi_{1}+\ldots+\xi_{n}$.

Theorem 1. In the class $\mathscr{F}_{1}$, the maximal value of $\operatorname{var} f(\xi)$ is attained at the function $f(x)=x_{1}+\ldots+x_{n}$. In other words, for any $f \in \mathscr{F}_{1}$,

$$
\operatorname{var} f(\xi) \leq \operatorname{var} S_{n}=\sum_{i=1}^{n} \sigma_{i}^{2} .
$$

Fernique (1981, Theorem 3.2) proved an inequality similar to (1.1) for $f \in \mathscr{F}_{1}$ convex. However, in that case $\xi$ is only assumed to be symmetrically distributed, i.e. for all $\epsilon_{i}= \pm 1$, the random vectors $\left(\epsilon_{i} \xi_{1}, \ldots, \epsilon_{n} \xi_{n}\right)$ have the same distribution (of course, this assumption holds if the $\xi_{i}$ are i.i.d. with a symmetric one-dimensional distribution). In contrast to Fernique's difficult proof, Theorem 1 can easily be obtained by induction.

Theorem 1 also has the following consequence: Denote by $M^{n}(\sigma)$ the family of all the

*To whom correspondence should be addressed.

1350-7265 (C) 1996 Chapman \& Hall 
product measures $\mu=\mu_{1} \otimes \ldots \otimes \mu_{n}$ on $\mathbb{\Omega}^{n}$ with given variance $\operatorname{var}(\mu)=\sigma^{2}$, where

$$
\operatorname{var}(\mu)=\sum_{i=1}^{n} \int_{\mathbb{R}}\left|x-\int_{\mathbb{R}} t \mathrm{~d} \mu_{i}(t)\right|^{2} \mathrm{~d} \mu_{i}(x) .
$$

Hence, with the above notation, $\operatorname{var}(\mu)=\operatorname{var} S_{n}$. Now, given a set $A \subset \mathbb{R}^{n}$ and $h>0$, denote by

$$
A^{h}=A+h B_{1}=\left\{x \in \mathbb{R}^{n}: d_{1}(a, x)<h, \quad \text { for some } a \in A\right\}
$$

the open $h$-neighbourhood of $A\left(B_{1}\right.$ is the open $\ell^{1}$-unit ball in $\left.\mathbb{R}^{n}\right)$. From Theorem 1 we obtain a solution to the isoperimetric problem with respect to the $\ell^{1}$-distance uniformly in the class $M^{n}(\sigma)$ controlled by the parameter $\sigma$.

Theorem 2. For any $h>0, \sigma>0$ and $p \in(0,1)$,

$$
\inf _{\mu \in M^{n}(\sigma)} \inf _{\mu(A) \geq p} \mu\left(A^{h}\right)=\left\{\begin{array}{ccc}
p, & \text { if } & h \leq \frac{\sigma}{\sqrt{p(1-p)}}, \\
1-\frac{p \sigma^{2}}{p h^{2}-\sigma^{2}}, & \text { if } & h \geq \frac{\sigma}{\sqrt{p(1-p)}} .
\end{array}\right.
$$

The first infimum in (1.2) is taken over all the $\mu \in M^{n}(\sigma)$, the second is taken over all the Borel sets $A$ of $\mu$-measure greater or equal to $p$. In particular, from Theorem 2, we have:

Corollary 3. Given $\sigma>0$ and $p \in(0,1)$, one can guarantee that $\mu\left(A^{h}\right)>p$ regardless of the dimension $n \geq 1$, regardless of the measure $\mu \in M^{n}(\sigma)$, and regardless of the set $A \subset \mathbb{R}^{n}$ of $\mu$ measure $p$, if and only if

$$
h>h(p, \sigma) \equiv \frac{\sigma}{\sqrt{p(1-p)}} .
$$

Otherwise, it is possible to have $\mu\left(A^{h}\right)=p$.

Equality in (1.2) is easy to obtain when $n=1$. Indeed, denote by $\delta_{x}$ the unit mass at the point $x \in$ R. If $h \leq h(p, \sigma)$, take

$$
\mu=p \delta_{0}+(1-p) \delta_{h(p, \sigma)}, \quad A=\{0\} .
$$

Then, $\operatorname{var}(\mu)=\sigma^{2}, A^{h}=(-h, h)$, so $\mu\left(A^{h}\right)=p=\mu(A)$. If $h \geq h(p, \sigma)$, take

$$
\mu=p \delta_{0}+q \delta_{x}+r \delta_{h}, \quad A=\{0\},
$$

with $r=p \sigma^{2} /\left(p h^{2}-\sigma^{2}\right), q=1-p-r, x=r h /(p+q)$. Then it is again easy to verify that $\operatorname{var}(\mu)=\sigma^{2}$, and that $\mu\left(A^{h}\right)=1-p \sigma^{2} /\left(p h^{2}-\sigma^{2}\right)$.

Since equality in (1.2) is attained when $n=1,(1.2)$ will not change if the $h$-neighbourhood is defined with respect to the $\ell^{2}$-distance, or, more generally, with respect to the $\ell^{\alpha}$ distance in $\mathbb{R}^{n}, 1 \leq \alpha \leq+\infty$. Indeed, the $\ell^{\alpha}$-unit ball $B_{\alpha}$ is larger than $B_{1}$, hence, $A+h B_{1} \subset A+h B_{\alpha}$, and therefore $\mu\left(A+h B_{1}\right) \leq \mu\left(A+h B_{\alpha}\right)$. Hence, the same inequality holds when one takes the second infimum in (1.2). But all the balls $B_{\alpha}$ coincide when $n=1$ (in which case equality in (1.2) is attained). 
For individual measures $\mu$ (for example, for those having finite exponential moments) there exist estimates for $1-\mu\left(A^{h}\right)$ which decrease exponentially when $h \rightarrow+\infty$ (see Talagrand 1994). For example, given $\sigma_{i}>0,1 \leq i \leq n$, let $\mu=\mu_{1} \otimes \ldots \otimes \mu_{n} \in M^{n}(\sigma)$, where $\mu_{i}=\left(\delta_{\sigma_{i}}+\delta_{-\sigma_{i}}\right) / 2, \sigma^{2}=\sigma_{1}^{2}+\ldots+\sigma_{n}^{2}$. Then, as shown in Talagrand (1994, Proposition 2.1.1., Theorem 2.4.1.) (see also Ledoux 1994, p. 24, for an extension to non-identical marginals), if $h>0, \mu(A)=p$, then

$$
\mu\left(A+h B_{1}\right) \geq 1-\frac{1}{p} \exp \left(-h^{2} / 4 \sigma^{2}\right) .
$$

When all the $\sigma_{i}=1$, the extremal sets minimizing $\mu\left(A^{h}\right)$, while $\mu(A)=p$ is fixed, are known, having been obtained by Harper (1966). If one minimizes $\mu\left(A^{h}\right)$ over all convex sets $A$, the situation changes considerably, and we are then dealing with a much more powerful concentration principle discovered by Talagrand $(1988 ; 1994)$. In particular, when all the $\sigma_{i}=1$, one has

$$
\mu\left(A+h B_{2}\right) \geq 1-\frac{1}{p} \exp \left(-h^{2} / 8\right) .
$$

In our case, since one is looking for a uniformly minimal value of $\mu\left(A+h B_{1}\right)$, it does not matter whether one considers convex sets or arbitrary sets, since the extremal $A=\{0\}$ is convex.

To complete this section, we give an inequality which is actually equivalent to the second part of (1.2). For non-empty sets $A, B \subset \mathbb{R}^{n}$, let $d_{1}(A, B)=\inf \left\{d_{1}(a, b): a \in A, b \in B\right\}$.

Corollary 4. For any $\mu \in M^{n}(\sigma)$, and any non-empty Borel sets $A, B \subset \mathbb{R}^{n}$,

$$
d_{1}(A, B) \leq \sigma \sqrt{\frac{1}{\mu(A)}+\frac{1}{\mu(B)}} .
$$

Let $\mu(A)>0, \mu(B)>0$ be such that $\mu(A)+\mu(B) \leq 1$. Then, choosing $B=\{h\}$ with $h$ equal to the right-hand side of (1.4), it is easily seen that equality in (1.4) is attained at the same measure $\mu$ and for the same set $A=\{0\}$ as the second inequality in (1.2).

\section{Proofs}

A statement slightly more general than Theorem 1 will actually be proved. Assume we have $n$ measurable spaces $\left(X_{k}, \Sigma_{k}\right)$ and $n$ measurable functions $h_{k}=h_{k}\left(x_{k}, y_{k}\right)$ defined on $X_{k} \times X_{k}, 1 \leq k \leq n$, and which vanish on the diagonal $x_{k}=y_{k}$. let $\xi_{k}$ be independent random variables with values in $X_{k}, 1 \leq k \leq n$, such that

$$
2 \sigma_{k}^{2}=\mathrm{E} h_{k}^{2}\left(\xi_{k}, \eta_{k}\right)<+\infty,
$$

where $\eta_{k}$ is an independent copy of $\xi_{k}$. Put $\xi=\left(\xi_{1}, \ldots, \xi_{n}\right)$. 
Lemma 5. Let $f$ be a measurable function defined on $X_{1} \times \ldots \times X_{n}$ such that

$$
\begin{array}{r}
|f(x)-f(y)| \leq \sum_{k=1}^{n} h_{k}\left(x_{k}, y_{k}\right), \\
\text { for } x=\left(x_{1}, \ldots, x_{n}\right), y=\left(y_{1}, \ldots, y_{n}\right) \in X_{1} \times \ldots \times X_{n} . \text { Then } \\
\operatorname{var} f(\xi) \leq \sum_{k=1}^{n} \sigma_{k}^{2} .
\end{array}
$$

Proof. This lemma is proved by induction on the dimension $n$. For $n=1$, and since 2var $f(\xi)=\iint(f(\xi)-f(\eta))^{2} \mathrm{~d} \mu(\xi) \mathrm{d} \mu(\eta),(2.2)$ is immediate. Assume now that (2.2) is true for $n$. Denote by $\mu_{n+1}$ the distribution of $\xi_{n+1}$, and by $P_{n}$ the distribution of the random vector $\left(\xi_{1}, \ldots, \xi_{n}\right)$, thus $P_{n+1}=P_{n} \otimes \mu_{n+1}$ is the distribution of $\left(\xi_{1}, \ldots, \xi_{n+1}\right)$. Let $f: X_{1} \times \ldots \times$ $X_{n+1} \rightarrow$ R satisfy (2.1). Now, fix $x_{n+1}$. Since the function $g\left(x_{1}, \ldots, x_{n}\right)=f\left(x_{1}, \ldots, x_{n}, x_{n+1}\right)$ satisfies (2.1), making use of the induction hypotheses and writing (2.2) for $g$, we obtain:

$$
\int g^{2} \mathrm{~d} P_{n} \leq\left(\int g \mathrm{~d} P_{n}\right)^{2}+\sum_{k=1}^{n} \sigma_{k}^{2} .
$$

The function $m\left(x_{n+1}\right)=\int g \mathrm{~d} P_{n}$ is well defined, measurable and as a function of one variable,

$$
\left|m\left(x_{n+1}\right)-m\left(y_{n+1}\right)\right| \leq h_{n+1}\left(x_{n+1}, y_{n+1}\right) .
$$

Thus $m$ satisfies (2.1), hence

$$
\int m^{2} \mathrm{~d} \mu_{n+1} \leq\left(\int m \mathrm{~d} \mu_{n+1}\right)^{2}+\sigma_{n+1}^{2} .
$$

Integrating (2.3) over $X_{n+1}$ (with respect to $\mu_{n+1}$ ), and taking into account (2.4), gives (2.2) for $f$. Lemma 5 and thus Theorem 1 are proved.

Proof of Theorem 2. Let $A \subset \mathbb{R}^{n}$ be such that $\mu(A) \leq p$. Since the function $f(x)=\inf _{a \in A} d_{1}(a, x)$ belongs to $\mathscr{F}_{1}$, we have, by Theorem 1, that var $f \leq \sigma^{2}$. In addition, $f \geq 0$ and $\mu(f=0) \geq p$. Note also that $A^{h}=\left\{x \in \Omega^{n}: f(x)<h\right\}$. To get (1.2), it just remains to appeal to the following result:

Lemma 6. For any $h>0, \sigma>0$ and $p \in(0,1)$,

$$
\sup P(\xi \geq h)=\left\{\begin{array}{ccc}
1-p, & \text { if } & h \leq \frac{\sigma}{\sqrt{p(1-p)}}, \\
\frac{p \sigma^{2}}{p h^{2}-\sigma^{2}}, & \text { if } & h \geq \frac{\sigma}{\sqrt{p(1-p)}},
\end{array}\right.
$$

where the supremum is taken over all non-negative random variables $\xi$ on a probability space $(\Omega, \mathscr{B}, P)$ such that $P(\xi=0) \geq p$ and $\operatorname{var} \xi \leq \sigma^{2}$. 
Proof. Denote by $\mathscr{L}(\xi)$ the distribution of $\xi$. The cases of equality in (2.5) were, in fact, already settled in Section 1. If

$$
h \leq h(p, \sigma), \quad \mathscr{L}(\xi)=p \delta_{0}+(1-p) \delta_{h(p, \sigma)},
$$

then $\operatorname{var}(\xi)=\sigma^{2}, P(\xi=0)=p, P(\xi \geq h)=1-p$. If

$$
h>h(p, \sigma), \quad \mathscr{L}(\xi)=p \delta_{0}+q \delta_{x}+r \delta_{h},
$$

where $r=p \sigma^{2} /\left(p h^{2}-\sigma^{2}\right), q=1-p-r, x=r h /(p+q)$, then, as easily verified, we have $\operatorname{var}(\xi)=\sigma^{2}, P(\xi=0)=p$, and $P(\xi \geq h)=r=p \sigma^{2}\left(p h^{2}-\sigma^{2}\right)$. So, one need only show that whenever $h \geq h(p, \sigma)$,

$$
P(\xi \geq h) \leq \frac{p \sigma^{2}}{p h^{2}-\sigma^{2}} .
$$

To prove this, we first show, following a suggestion by M. Talagrand, that in (2.6) it suffices to consider only those $\xi$ whose distribution is of the type $\mathscr{L}(\xi)=p_{0} \delta_{0}+p_{1} \delta_{x}+p_{2} \delta_{h}$, for some $0 \leq x<h$. Then, keeping $p_{2}$ constant, we maximize the functional $J=p_{2}$ over all $p_{0} \geq p$ and $x \in[0, h)$ such that $\operatorname{var}(\xi) \leq \sigma^{2}$.

Note first that in (2.6), $\xi$ can be replaced by $\eta=\min (\xi, h)$ since $P(\eta \geq h)=P(\xi \geq h)$, while $\operatorname{var}(\eta) \leq \operatorname{var}(\xi) \leq \sigma^{2}$ ( $\eta$ is a Lipschitz function of $\xi: \eta=f(\xi)$, where $f(t)=$ $\min (t, h))$. Then, let $\xi$ take values in $[0, h]$, have distribution $\nu$, and assume that $\nu(0, h)>0$. Then, $\nu$ can (uniquely) be written as

$$
\nu=p_{0} \delta_{0}+p_{1} \lambda+p_{2} \delta_{h}
$$

where the distribution $\lambda$ is concentrated in $(0, h)$. Let $\xi_{1}$ be a random variable whose distribution is $\lambda$, and let $x=\mathrm{E}\left(\xi_{1}\right)$. Then

$$
\begin{aligned}
\operatorname{var}(\xi) & =p_{1} \mathrm{E}\left(\xi_{1}^{2}\right)+p_{2} h^{2}-\left(p_{1} x+p_{2} h\right)^{2} \\
& =p_{1} \operatorname{var}\left(\xi_{1}\right)+p_{1}\left(1-p_{1}\right) x^{2}-2 p_{1} p_{2} h x-p_{2} h^{2} .
\end{aligned}
$$

Therefore, given the mean value $x, \operatorname{var}(\xi)$ is minimal if and only if $\lambda=\delta_{x}$, when var $\left(\xi_{1}\right)=0$. Thus, $\xi$ can be replaced in (2.6) by a random variable $\eta$ which takes three values, 0 , $x$ and $h$.

So let us assume that $\mathscr{L}(\xi)=p_{0} \delta_{0}+p_{1} \delta_{x}+p_{2} \delta_{h}, \quad 0 \leq x<h, p_{0} \geq p, p_{1}, p_{2} \geq 0$, $p_{0}+p_{1}+p_{2}=1$. Again, $J=p_{2}$ is constant. By simple algebra, and for fixed $p_{0}, p_{1}, p_{2}$, the minimal value of

$$
\operatorname{var}(\xi)=\left(p_{1} x^{2}+p_{2} h^{2}\right)-\left(p_{1} x+p_{2} h\right)^{2},
$$

as a function of $x$ in $(0, h)$, is attained at

$$
x=\frac{p_{2}}{p_{0}+p_{2}} h .
$$


For this value of $x$, we find

$$
\begin{aligned}
\operatorname{var}(\xi) & =\left(p_{1} \frac{p_{2}^{2}}{\left(p_{0}+p_{2}\right)^{2}} h^{2}+p_{2} h^{2}\right)-\left(p_{1} \frac{p_{2}}{p_{0}+p_{2}} h+p_{2} h\right)^{2} \\
& =\left[\frac{p_{2}}{\left(p_{0}+p_{2}\right)^{2}}\left(p_{1} p_{2}+\left(p_{0}+p_{2}\right)^{2}\right)-\frac{p_{2}^{2}}{\left(p_{0}+p_{2}\right)^{2}}\left(p_{1}+\left(p_{0}+p_{2}\right)\right)^{2}\right] h^{2} \\
& =\frac{p_{2}}{\left(p_{0}+p_{2}\right)^{2}}\left[p_{1} p_{2}+\left(p_{0}+p_{2}\right)^{2}-p_{2}\right] h^{2} \\
& =\frac{p_{0} p_{2}}{p_{0}+p_{2}} h^{2} .
\end{aligned}
$$

Now, we have to maximize $J=p_{2}$ under the condition

$$
\operatorname{var}(\xi)=\frac{p_{0} p_{2}}{p_{0}+p_{2}} h^{2} \leq \sigma^{2} .
$$

From (2.7), when $p_{0}$ decreases, $\operatorname{var}(\xi)$ also decreases, while $J=p_{2}=1-p_{0}-p_{1}$ increases ( $p_{1}$ is fixed). Hence, to conclude, it is enough to consider only the case $p_{0}=p$. The possible maximal value $p_{2}=1-p$ satisfies (2.7) if and only if $p(1-p) \leq \sigma^{2} / h^{2}$, that is, if and only if $h \leq h(p, \sigma)$. Otherwise, if $h>h(p, \sigma)$, or even if $h=h(p, \sigma)$, the maximal value of $J=p_{2}$ is, according to (2.7), the value which satisfies

$$
\frac{p p_{2}}{p+p_{2}} h^{2}=\sigma^{2}
$$

The only solution to this equation is given by

$$
p_{2}=\frac{p \sigma^{2}}{p h^{2}-\sigma^{2}} .
$$

Lemma 6 follows.

Proof of Corollary 4. Let $p=\mu(A), q=\mu(B)$. If $p=0$ or $q=0$, there is nothing to prove. If $p+q>1$, then $A \cap B \neq \emptyset$, so $d_{1}(A, B)=0$. Thus, we need consider only the case $p+q \leq 1$. Let $p, q>0, p+q \leq 1$, and assume $A \cap B=\emptyset$. Note that

$$
h \equiv \sigma \sqrt{\frac{1}{p}+\frac{1}{q}} \geq h(p, \sigma) .
$$

Therefore, by (1.2),

$$
1-\mu\left(A^{h}\right) \leq \frac{p \sigma^{2}}{p h^{2}-\sigma^{2}}=q,
$$

and again by (1.2), for all $h_{1}>h, 1-\mu\left(A^{h_{1}}\right)<q$. Hence, $B \cap\left(A^{h_{1}} \backslash A\right) \neq \emptyset$, and therefore, $d_{1}(A, B) \leq h_{1}$. Letting $h_{1} \rightarrow h$ completes the proof. 


\section{Acknowledgements}

Many thanks are due to M. Talagrand who provided an argument significantly simplifying the original proof of Lemma 6 . The research of S.G. Bobkov was supported partly by ISF grants NZX000 and NZX300. The research of C. Houdré was supported partly by an NSF Mathematical Sciences Postdoctoral Fellowship.

\section{References}

Fernique, X. (1981) Régularité de fonctions aléatoires non gaussiennes. Lecture Notes in Math.976, pp. 2-74. New York: Springer-Verlag.

Harper, H. (1966) Optimal numbering and isoperimetric problems on graphs. J. Combin. Theory, 1, $385-393$.

Ledoux, M. (1994) Isoperimetry and Gaussian analysis. École d'été de Probabilités de Saint-Flour.

Talagrand, M. (1988) An isoperimetric theorem on the cube and the Khinchine-Kahane inequalities. Proc. Amer. Math. Soc., 104, 905-909.

Talagrand, M. (1994) Concentration of measure and isoperimetric inequalities in product spaces. Preprint.

Received July 1995 and revised January 1996 